\title{
Higher Vibrational Modes In RR Lyrae Stars
}

\author{
S. A. Glasner ${ }^{1}$, J. R. Buchler ${ }^{2}$, \\ ${ }^{1}$ The Racah Institute of Physics, Jerusalem, Israel, \\ ${ }^{2}$ Physics Department, University of Florida, Gainesville, Florida, USA
}

\begin{abstract}
A simple method for computing the full spectrum of the linear (nonadiabatic) radial modes is implemented and tested on RR Lyrae models. The growthrates of the vibrational modes display unexpected, but physically correct undulations as a function of period, with the $8^{\text {th }}$ through $10^{\text {th }}$ overtones almost unstable. Caution must be exercised when only a small number of meshpoints is used, or when the meshpoints are not well distributed, as some of these overtones may become unstable, clearly an artifact of the differencing. The role that such unstable high overtones can play in hydrodynamic calculations is demonstrated.
\end{abstract}

The lowest order vibrational modes are frequently computed with the Castor (1971) method. Unfortunately this approach fails for the higher vibrational modes as well as for the thermal modes. To overcome this difficulty here, we transform the linearized system of hydrodynamics into a standard eigenvalue problem of the form $\mathbf{A} \cdot \mathbf{z}=\sigma \mathbf{z}$, where $\mathbf{A}$ is a $3 N \times 3 N$ constant matrix and $\mathbf{z} \in \mathbf{R}^{3 N}$ is the vector of components $\left\{\delta R_{k}, \delta u_{k}, \delta T_{k}\right\}, k=1, N$. To achieve this form we have made use of the continuity equation to replace $p d V / d t$ in the energy equation in terms of the velocity $u$. Standard procedures exist for finding the eigenvalues of such a general (non Hermitean) real matrix.

As an application we have computed the spectrum of vibrational modes for a few RR Lyrae models, viz. $0.6 M_{\odot}, 60 L_{\odot}$ with $T_{\text {eff }}$ in the range $6800 \mathrm{~K}$ to $7600 \mathrm{~K}$. The composition in all models is $X=0.7$ and $Z=0.001$. Because of the sensitivity of the growthrates to zoning we have used up to 1400 zones. The Figure displays the resultant relation between the frequencies $f_{k}$ and the relative growthrates $\eta_{k}=2 \kappa_{k} / f_{k}$ for the lowest vibrational modes in the $T_{\text {eff }}=7300 \mathrm{~K}$ model which is typical of all the RR Lyrae models. The surprising result is that the growthrates do not level off with increasing mode number, but that instead they show an undulatory behavior. The first excursion is in fact quite substantial with the result that the $8^{\text {th }}$ through $10^{\text {th }}$ overtones are only very weakly damped. The undulations are a result of the phase relationship between $\delta p$ and $\delta \rho$ which varies with the spatial structure for the successive overtones. The first large decrease in driving (culminating with the $4^{\text {th }}$ overtone) is due to an adverse phase relationship which occurs in the He partial ionization region. For the higher modes the partial He ionization region no longer contributes any driving, which comes entirely from nonadiabatic effects in the partial $\mathrm{H}$ ionization region. This driving is particularly efficient for the $9^{\text {th }}$ overtone. 
The existence of marginally stable overtones suggests that it may be possible to detect these frequencies in RR Lyrae if they are stochastically excited (in a fashion similar to that of the solar p-modes). This raises the exciting prospect of being able to do astro-seismology on the classical variable stars.

Figure: $\quad \eta v s$ frequency for an RR Lyr model with $T_{\text {eff }}=7300 \mathrm{~K}$

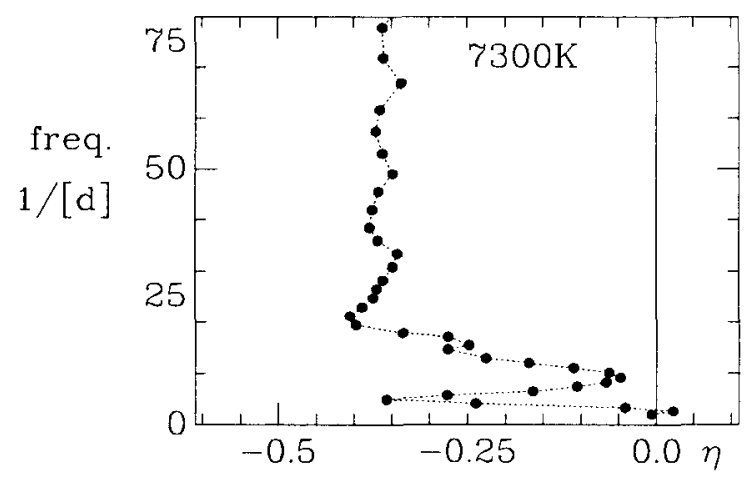

Our study reveals a strong sensitivity of the linear growthrates to the numerical mesh, especially for the higher vibrational modes. For unfortunate choices of the numerical mesh the decrease of stability around overtones $8-10$ can give rise to unstable modes $(\eta>0)$ and to unphysical effects in the numerical hydrodynamic computations.

In order to illustrate the phenomenon we have constructed models with $T_{\text {eff }}=$ $7300 \mathrm{~K}$ this time with a coarser grid of 120 zones, suitable for hydrodynamical computations. One model, e.g. has 30 equally spaced mass zones up to $11,000 \mathrm{~K}$, followed by geometrically increasing zones up to $2 \mathrm{MK}$. Another model has again 30 mass zones up to $11,000 \mathrm{~K}$, but with a geometric progression of the mass zone, followed by a geometrically increasing mesh as for the first model. In the two models the first 5 overtones have almost the same properties as those of the finely resolved model. The spectrum of eigenvalues for the higher overtones also has a qualitatively similar behavior. However, it is interesting that the $9^{\text {th }}$ overtone for the second model is actually vibrationally unstable, while it remains stable in the first model.

Since only the first overtone is linearly unstable in the first model the latter exhibits only stable first overtone limit cycle pulsations. On the other hand the nonlinear behavior of the second model which is linearly unstable in two modes is different. When the model is destabilized with a more or less arbitrary kick the pulsations contain a strong admixture of the linearly unstable $9^{\text {th }}$ overtone which persist for a long time and show up as wiggles with a frequency $f_{9} \approx 3.5 f_{1}$. In addition, there are now at least two stable limit cycles ( $1^{\text {st }}$ overtone and $9^{\text {th }}$ overtone) with a unstable mixed mode state. These results therefore suggest that some caution needs to be exercised when an initial model is constructed to ensure that the differencing does not introduce unphysical unstable overtones and unphysical attractors.

This work has been supported by NSF. 